Check for updates

Cite this: Chem. Sci., 2019, 10, 4502

๑ All publication charges for this article have been paid for by the Royal Society of Chemistry

Received 4th January 2019

Accepted 11th March 2019

DOI: $10.1039 / c 9 s c 00059 c$

rsc.li/chemical-science

\section{Identification of selective protein-protein interaction inhibitors using efficient in silico peptide-directed ligand design $\uparrow$}

\author{
Andrew M. Beekman, (D) *a Marco M. D. Cominetti, ${ }^{\text {a }}$ Samuel J. Walpole, ${ }^{\text {a }}$ \\ Saurabh Prabhu, ${ }^{a}$ Maria A. O'Connell, ${ }^{a}$ Jesus Angulo a and Mark Searcey (DD *ab
}

\begin{abstract}
The development of protein-protein interaction (PPI) inhibitors with therapeutic value is of increasing importance as the first clinical agent has now been approved, but PPIs remain difficult targets for the development of small molecule ligands. This article describes a highly efficient approach to the development of inhibitors of the p53/hDMX or hDM2 interaction that involves the design of small molecules in silico based upon a peptide/protein structure. The process for molecule design, starting from a virtual library of just over 1200 fragments, led to the eventual synthesis of twenty compounds, of which ten bound to either hDM2, hDMX or both in in vitro binding assays. This $50 \%$ success rate is extremely efficient compared to traditional high throughput screening. The identification of two selective $h D M X$ inhibitors from twenty compounds highlights this efficiency as, to date, only two other $h D M X-$ selective agents exist in the literature. Preliminary biological studies show that $20 \%$ of the compounds identified have cellular activity and activate downstream pathways associated with p53 activation.
\end{abstract}

\section{Introduction}

Targeting the hDM2 (human double minute 2) p53 proteinprotein interaction (PPI) has been a paradigm for PPI inhibitor development in cancer since the discovery of nutlin-3, which is currently undergoing clinical trials. ${ }^{1,2}$ Although there are many studies of p53- $h \mathrm{DM} 2$ interaction inhibitors ranging from natural products to designed small molecules, the scope of this promising strategy has been severely limited by the activity of $h \mathrm{DMX} .^{3}$ Despite the highly homologous nature of $h \mathrm{DMX}$ to $h \mathrm{DM} 2$ the two proteins do not perform redundant roles. ${ }^{4,5} h \mathrm{DMX}$ can prevent p53 activity by sequestering it, but $h \mathrm{DMX}$ also enhances the ligase activity of $h \mathrm{DM} 2$ towards p53 by forming $h \mathrm{DM} 2 / h \mathrm{DMX}$ dimers. ${ }^{6}$ The overexpression of one or both of these proteins results in the loss of p53 activity in cells. ${ }^{7}$ Therefore, dual $h$ DM2 $h$ DMX inhibitors have become highly desirable.

The development of $h \mathrm{DMX}$ selective molecules is also of significant importance. It has been shown that direct inhibition of $h \mathrm{DM} 2$ leads to p53 dependent toxicity in healthy cells., Additionally, the non-redundant roles and common mutual exclusivity in cancers suggest selective compounds are useful. ${ }^{10}$ There are currently no small molecules that modulate $h \mathrm{DMX} / \mathrm{p} 53$

${ }^{a}$ School of Pharmacy, University of East Anglia, Norwich Research Park, Norwich, Norfolk, NR47TJ, UK.E-mail: A.Beekman@uea.ac.uk; M.Searcey@uea.ac.uk ${ }^{b}$ School of Chemistry, University of East Anglia, Norwich Research Park, Norwich, Norfolk, NR47TJ, UK

† Electronic supplementary information (ESI) available: Experimental procedures, spectra and processed biophysical assay data. See DOI: 10.1039/c9sc00059c and exhibit both cell and animal efficacy. ${ }^{11}$ Two compounds have been highlighted which modulate $h \mathrm{DMX} / \mathrm{p} 53$ selectively, but are unsuitable for clinical development. ${ }^{\mathbf{8 , 9}, 12}$ Therefore, there is still a demand for chemical probes or leads that are selective for $h \mathrm{DM} 2$, selective for $h \mathrm{DMX}$ or dual $h \mathrm{DM} 2 h \mathrm{DMX}$ inhibitors. The p53 binding domains of $h \mathrm{DM} 2$ and $h \mathrm{DMX}$ display high structural homology, but differences exist which could be exploited. ${ }^{7,10}$

Several approaches to the identification of inhibitors of the p53- $h$ DM2 and p53- $h$ DMX interaction have been described. Natural products such as chlorofusin can be utilised as starting points for design. ${ }^{13}$ Oligobenzamide analogues that mimic the interactions of the helical peptide have been described ${ }^{\mathbf{1 4 , 1 5}}$ and helical peptides have been stabilised and shown to have cellular activity through the introduction of stapling groups. ${ }^{16}$ Both high throughput screening and computational design against the $h \mathrm{DM} 2$ target have had varying degrees of success with hit rates for screening of around $0.02 \%$.

We believe these challenging PPIs are excellent targets for the exemplification of in silico peptide directed binding, a new methodology for identifying selective PPI modulators. Using copper(I) catalysed azide-alkyne cycloaddition (CuAAC) click chemistry and taking a small commercially available library of 896 alkynes and 214 azides would require the synthesis of 191744 compounds to screen all possible compounds. Using these compounds as fragments for screening would need high resolution NMR with ${ }^{15} \mathrm{~N}$ labelled protein or high throughout crystallography, relatively specialised techniques. Our approach has been to exploit the peptidic dual inhibitor Ac-Phe-Met-Aib-Pmp-6-Cl-Trp-Glu-Ac $\mathrm{A}_{3} \mathrm{c}$ Leu-NH $\mathrm{NH}_{2}(\mathbf{1})$ to develop small molecule probes that target $h \mathrm{DM} 2{ }^{17}$ 
and $h \mathrm{DMX} .^{18}$ Briefly, the crystal structure of $\mathbf{1}$ bound to both proteins is known ( $h$ DMX: PDB ID 2GV2, hDM2: PDB ID 3FEA). The sequence of the high affinity peptide binder is used to generate two smaller peptides with reactive azide and alkyne terminals (Fig. 1). ${ }^{\mathbf{1 9}}$ In silico screening of the small molecule fragments then allows the identification of peptide/small molecule hybrids with restored affinity for the target site. The restoration of binding affinity implies that the small molecule fragment in some way emulates the peptide section it has replaced. The small molecule portion of the hybrid hits are then combined through in silico click chemistry and rescreened to identify potential small molecules with high affinity for the target site. Computational modelling is used to perform the entire peptide directed binding process, identifying small molecules to be prepared. This use of virtual design in peptide directed binding further improves the rapid and economic nature of this process, identifying compounds not highlighted by experimental peptide directed binding. ${ }^{19}$ Herein, we demonstrate that this approach led to the synthesis of twenty small molecules of which ten bound to $h \mathrm{DM} 2$ or $h \mathrm{DMX}$, representing a 50\% success rate. Furthermore, one compound was selective for $h \mathrm{DMX}$ both in vitro and in tissue culture, outperforming previously reported selective modulators.

\section{Results}

\section{In silico peptide directed binding identifies candidate small molecule $h \mathrm{DM} 2 / \mathrm{X}$ binders}

Peptide 1 binds with high affinity to both $h \mathrm{DM} 2$ and $h \mathrm{DMX}$, which allows its exploitation to develop small molecule dual

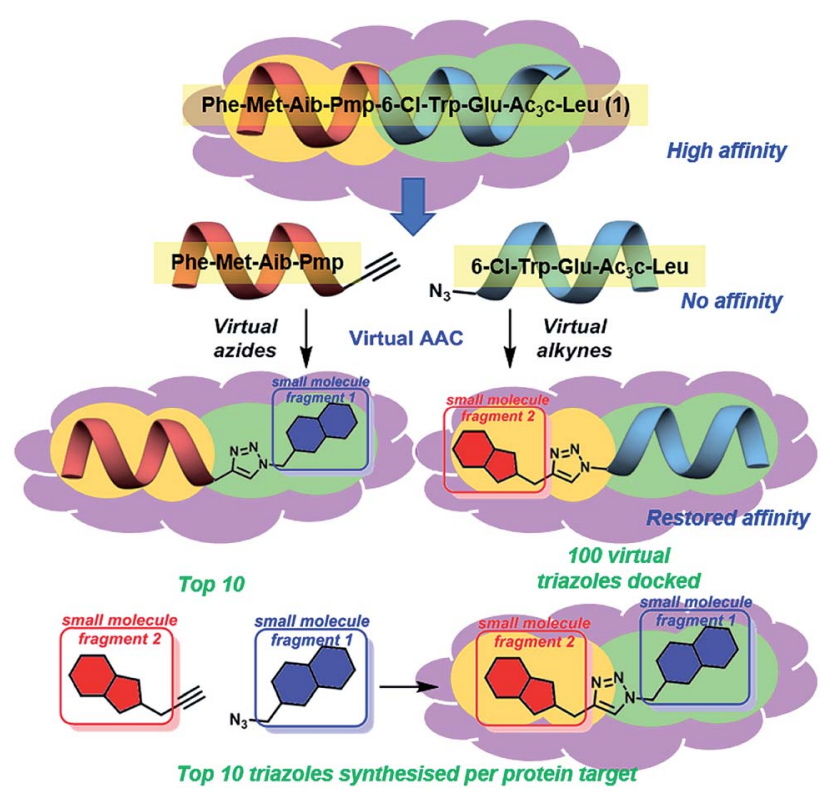

Fig. 1 Concept of peptide directed binding utilised in this work with in silico methods to improve the rapid and economic nature of finding new PPI modulators. Peptide 1 binds to $h D M 2 / X$, and is separated into two smaller peptides with no appreciable binding despite having two key residues each. Azide-alkyne cycloaddition (AAC) identifies small molecule fragments which restore binding affinity. The identified small molecule fragments can be combined to generate a small molecule substitute for the initial peptide. inhibitors as both chemical probes and potential therapeutic lead molecules. In order to identify small molecules to be synthesised and evaluated against $h \mathrm{DM} 2$ and $\mathrm{h} D \mathrm{MX}$ the crystal structures for the peptide 8-mer 1 bound to $h \mathrm{DM} 2$ (PDB ID: $2 \mathrm{GV} 2)^{17}$ and $h$ DMX (PDB ID: $\left.3 \mathrm{FEA}\right)^{\mathbf{1 8}}$ were edited to identify small molecule fragments that emulate a section of the 8 mer. The peptide was split, Phe-Met-Aib-Pmp-propargyl glycine and azidoacetamide-6-Cl-Trp-Glu-Ac ${ }_{3}$ c-Leu (Fig. 1), allowing two key binding residues to be present in both smaller peptides (Phe and Pmp, 6-Cl-Trp and Leu). The site left vacant by the removed peptide fragment represents a more tractable binding site for a small molecule. Covalent docking was then performed simulating the Huisgen 1,3-dipolar cycloaddition (see ESI, $\dagger$ pg 6), with the commercially available library of alkynes or azides (ESI, $\uparrow$ pg 12). The semi-peptide was held in place with flexibility in the reactive terminal and the final residue, allowing for the possibility that the added small molecule fragment is held in the correct orientation for binding. The results were scored and ranked $^{\mathbf{2 0}}$ and the top ten azides and top ten alkynes that were synthetically viable were used to generate one hundred virtual triazoles. The generated triazoles were then induced-fit docked to the protein, scored and ranked to identify the top ten small
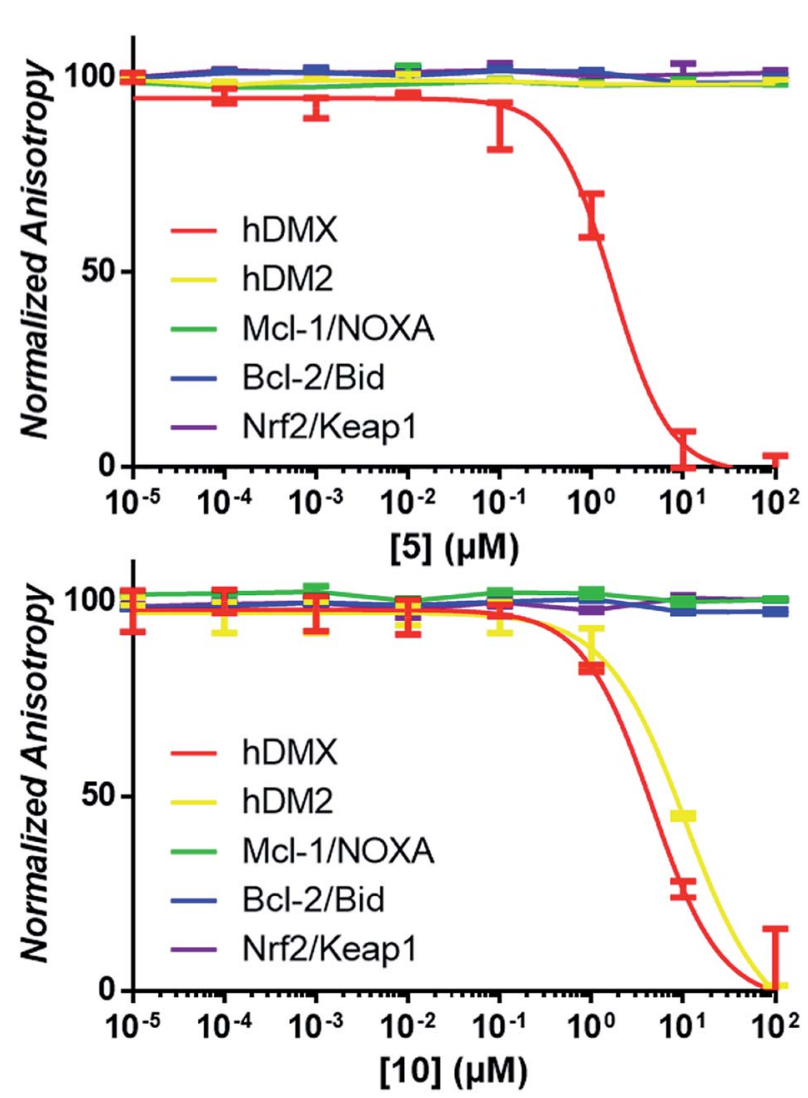

Fig. 2 Examples of competitive-displacement assays of small molecules towards a selection of PPIs. Compounds 5 and 10 used to exemplify the titration of compounds towards the $h \mathrm{DMX} / \mathrm{p} 53, h \mathrm{DM} 2 /$ p53, Mcl-1/NOXA, Bcl-2/Bid and Nrf2/Keap1 interactions. Dose response curves can be seen for 5 towards hDMX, and for 10 towards $h \mathrm{DMX}$ and $h \mathrm{DM} 2$, with no competitive binding towards any other PPIs observed. 
molecules that bound in silico with high affinity to each protein and that would be synthesised (Fig. 1). ${ }^{21}$ The identified triazole small molecules are likely to bind with the same binding elements as identified by the hybrid, but may sit in a slightly altered orientation to allow for tighter binding. This process was carried out for both $h \mathrm{DM} 2$ and $h \mathrm{DMX}$ and as such twenty small molecule triazoles were synthesised following published procedures with $\mathrm{CuSO}_{4}$ and sodium ascorbate in a ${ }^{t} \mathrm{BuOH} / \mathrm{H}_{2} \mathrm{O}$ mix, followed by purification by reverse phase HPLC (see ESI $\dagger$ pg. 9 for all synthesised compounds). ${ }^{13}$

\section{Identified small molecules selectively modulate the p53- hDM2/X PPIs}

It was expected that some of these molecules would display selectivity for the target used in their design, but inevitably dual modulators would be identified due to the homogeneity of the $h \mathrm{DM} 2 / \mathrm{X}$ binding sites and the use of the same peptide 1 . The synthesised compounds were first analysed with competitive fluorescence anisotropy (FA) assays. A FAM tagged p4 peptide $\mathrm{p}^{22,23}$ was used as the fluorescently tagged peptide for both $h \mathrm{DM} 2$, reported previously, ${ }^{13}$ and $h \mathrm{DMX}$. The wild-type p53 peptide (residues 15-27) was employed as a positive control and an indication that the FA assays were performing adequately. Of the twenty compounds synthesised, 50\% demonstrated binding in the FA assays to either $h \mathrm{DM} 2$ or $h \mathrm{DMX}$, with $\mathrm{IC}_{50}$ values less than $100 \mu \mathrm{M}$ (Table 1). Strikingly, one compound displayed significant selectivity for $h \mathrm{DMX}$ over $h \mathrm{DM} 2(5)$, while overall $40 \%$ of compounds designed for $h \mathrm{DMX}$ modulated the $h \mathrm{DMX}$ interaction (2-5). Two of these compounds, 2 and 3, had an $\mathrm{IC}_{50}$ in the tens of nanomolar range. Of the compounds designed for $h \mathrm{DM} 2$, $50 \%$ demonstrated the ability to modulate the $h \mathrm{DM} 2$ PPI (6-10), with three showing selectivity for $h \mathrm{DM} 2$ over $h \mathrm{DMX}$ (7-9). Interestingly, one of the compounds designed for $h \mathrm{DM} 2$ (11) had affinity for $h \mathrm{DMX}$ but no appreciable binding to $h \mathrm{DM} 2$. To confirm selectivity, these compounds were analysed in other PPI FA assays routinely utilised in our lab. Pleasingly, compounds 211 demonstrated no appreciable binding at $100 \mu \mathrm{M}$ towards the PPIs Mcl-1/Noxa, Bcl-2/Bid ${ }^{19}$ and Nrf2/Keap1, ${ }^{24}$ despite similarities to compounds reported to bind to these PPIs (Fig. 2).

The compounds that exhibited binding in the FA assay were validated with differential scanning fluorimetry (DSF). ${ }^{25}$

Table $1 \quad I_{50}$ values for inhibition of the binding of FAM-p4 to $h \mathrm{DM} 2$ and $h \mathrm{DMX}$ of small molecules. ${ }^{a}$ A green background indicates the compound was designed for and was selective to the same protein. Yellow indicates the compound is a dual inhibitor. A red background indicates the compound was designed for one protein but was selective for the other protein

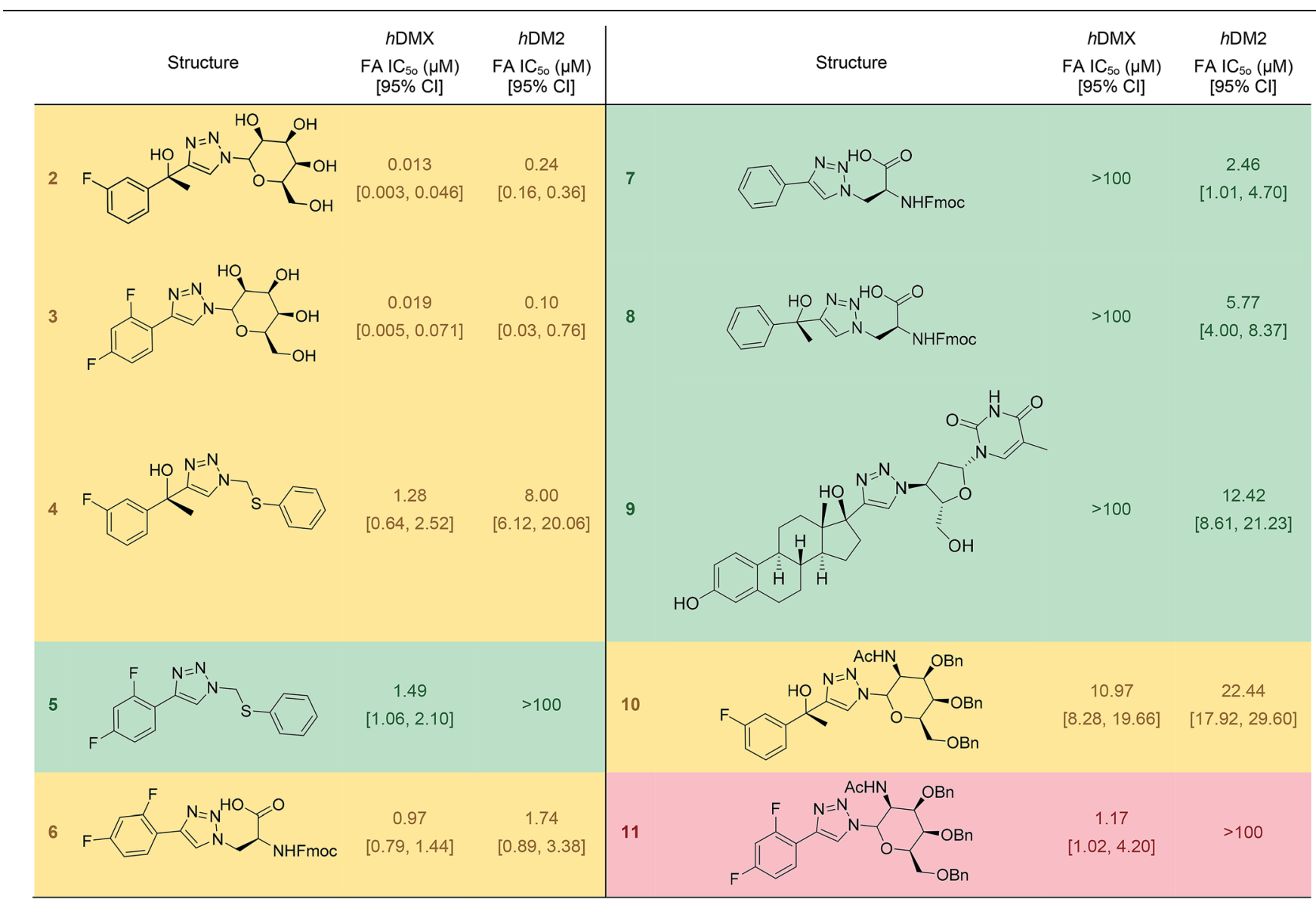

${ }^{a} \mathrm{IC}_{50}$ values determined by non-linear regression of at least three independent experiments (see ESI, $\dagger$ pg 10). Errors are 95\% confidence intervals (CI). Fmoc, 9-fluorenylmethylcarbonyl. 
Compounds displayed the ability to increase the melting temperature of proteins compared to a vehicle control (see ESI, $\dagger$ pg. 41). ${ }^{26}$ Thermal stability was demonstrated using the hydrophobic dye Sypro orange, mimicking the observed FA assay results (see ESI, $\dagger$ pg. 40).

\section{Use of STD NMR indicates that compounds bind in the p53 binding site of $h \mathrm{DM} 2 / \mathrm{X}$}

To provide structural details of the binding of these compounds in the p53 binding site, we employed an STD NMR initial growth-rates approach to identify the binding mode and the interactions with the binding grooves of $h \mathrm{DM} 2$ and $h \mathrm{DMX} \cdot{ }^{27-29}$ Compound 4 was chosen for analysis by STD NMR because of its solubility in aqueous buffer and its relatively weak binding to both $h \mathrm{DM} 2$ and $h \mathrm{DMX}$, allowing the binding kinetics to fall within the fast-exchange conditions necessary for STD NMR. This criteria precludes tighter binders (2 \& 3) from being analysed with this technique. Compound 4 displayed STD signals in the presence of either $h \mathrm{DM} 2$ or $h \mathrm{DMX}$, indicating binding to both proteins.

Additionally, the ligand binding epitope of compound 4 was mapped using the STD-NMR initial growth rates method (ESI, $\dagger$ pg 15). In order to gain some structural information docking calculations were used to identify a binding pose. We were able to validate the binding pose within the $h \mathrm{DM} 2$ binding groove using CORCEMA-ST, ${ }^{30}$ which calculates theoretical STD intensities based on a given $3 \mathrm{D}$ model complex. The NOE R-factor between the experimental and theoretical STD intensifies was 0.17 , indicating good agreement. Interestingly, preliminary CORCEMA-ST trials of $\mathbf{4}$ binding to $h$ DMX suggested the formation of oligomers. Dimerization induced by structurally similar compounds has been observed previously, ${ }^{8}$ and suggests a possible selective binding mode which could be exploited for $h \mathrm{DMX}$ modulation. Fig. 3 displays the binding pose of 4 to $h$ DM2 demonstrating tight binding of the fluorinated aryl ring in the pocket vacated by chlorotryptophan of 1 . This STD/CORCEMA-ST validated binding pose, along with the binding affinities determined by the competitive fluorescence anisotropy assay indicate these compounds are binding in the p53 binding groove. The identified binding epitope places the fluorine atom in the same pocket as that occupied by the chlorine atom of the ClTrp in 1. This same pocket also exists in the $h \mathrm{DMX}$ binding site and may explain the improved binding of the fluoroaryl group (2, 10 \& 11 compared to $\mathbf{S 8 ,}$ S2 \& S9).

\section{Compounds inhibit cancer cell growth in a selective fashion}

Compounds that had activity in the FA assay were examined for their ability to inhibit cell growth and affect metabolism in an array of cancer cell lines that demonstrate varying expressions of the p53 and $h \mathrm{DM} 2 / \mathrm{X}$ proteins. The human cancer cell lines A549, ${ }^{31}$ HCT116 ${ }^{3,31}$ and MCF-7 ${ }^{31}$ express both $h \mathrm{DM} 2$ and $h$ DMX, SJSA-1 is $h$ DM2 dependent ${ }^{\mathbf{1 1}}$ and JEG3 is $h$ DMX dependent. $^{3}$ All cell lines express wild type p53. Gratifyingly, $10 \%$ of the prepared compounds demonstrated the ability to inhibit cell growth. Compound 10, which was shown to bind to both

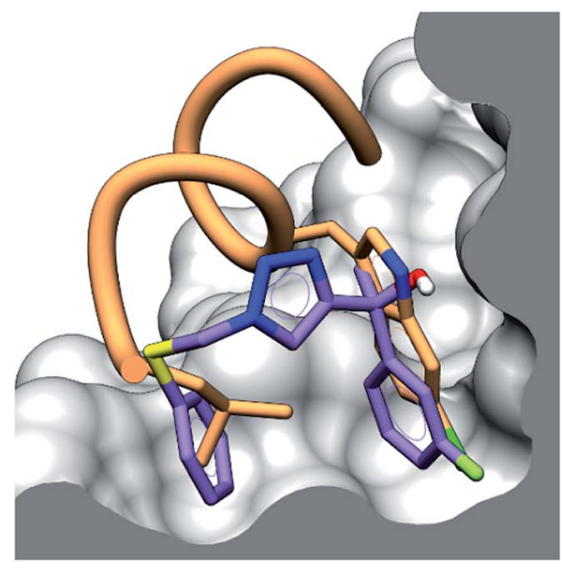

Fig. 3 Binding pose of 4 (purple) to $h D M 2$ in the p53 binding site determined by CORCEMA-ST, with an overlay of peptide 1 (orange) showing the binding residues of Leu and Cl-Trp. The surface of $h \mathrm{DM} 2$ is cut along the plane of the centroid of the aromatic rings of compound 4 .

$h \mathrm{DM} 2$ and $h \mathrm{DMX}$, had activity towards cell lines that are dependent on $h \mathrm{DMX}$, dependent on $h \mathrm{DM} 2$ or dependent on both, as expected (Table 2). Compound 5, which showed selectivity for $h \mathrm{DMX}$ over $h \mathrm{DM} 2$, demonstrated activity towards the $h \mathrm{DMX}$ dependent JEG3, but not towards the $h \mathrm{DM} 2$ dependent SJSA-1, a further suggestion of selectivity of $\mathbf{5}$ for $h \mathrm{DMX}$. The remaining compounds did not demonstrate activity in the MTS assay, perhaps due to issues with cell penetration. Alterations to the in silico screening process are currently underway to increase the output of compounds with desirable characteristics, such as cell permeability.

\section{Compounds induce events downstream of p53 activation}

To ascertain whether compounds 5 and $\mathbf{1 0}$ were inducing p53 related cell death we examined the induction of apoptosis with a caspase 3/7 activation assay. A549 and JEG-3 cells were treated with vehicle (DMSO), positive control (staurosporine), $\mathbf{5}$ and $\mathbf{1 0 .}$ Cleavage of the DEVD sequence was monitored to observe caspase $3 / 7$ activity. Activation of caspase $3 / 7$ was observed for both 5 and 10 in line with the observed $\mathrm{IC}_{50}$ values (Fig. 4A). The transcriptional activation of targets downstream of p53 was examined with quantitative PCR (qPCR). ${ }^{3}$ JEG-3 cells were treated with vehicle control, $\mathbf{5}$ and $\mathbf{1 0}$ for $6 \mathrm{~h}$ at $37^{\circ} \mathrm{C}$, followed by RNA isolation, reverse transcription, and qPCR analysis of the obtained cDNA using the primers of downstream p53 targets p53, $h \mathrm{DM} 2, \mathrm{p} 21$ and MIC-1. Both compounds 5 and 10 significantly increased the expression of each of the target genes when compared to the vehicle control (Fig. 4B).

Virtual peptide directed binding was employed to identify new modulators of $\mathrm{p} 53 / h \mathrm{DM} 2$ and $\mathrm{p} 53 / h \mathrm{DMX}$. This use of computational peptide directed binding represents a complementary method to identify PPI modulators in a rapid and economic manner. Twenty compounds were prepared, ten designed for $h \mathrm{DM} 2$ and ten designed for $h \mathrm{DMX}$, and $50 \%$ of the compounds were found to modulate the PPI. This hit rate is a significant improvement compared to recent studies which 
Table 2 Cell growth inhibition of compounds which demonstrated activity towards cell line which are dependent on $h \mathrm{DM} 2, h \mathrm{DMX}$ or both, demonstrated by the MTS assay ${ }^{a}$

\begin{tabular}{llllll}
\hline & SJSA-1 IC $_{50}(\mu \mathrm{M})$ & $\mathrm{A549} \mathrm{IC}_{50}(\mu \mathrm{M})$ & $\mathrm{HCT116}_{\text {IC }}(\mu \mathrm{M})$ & MCF-7 IC $_{50}(\mu \mathrm{M})$ & $\mathrm{JEG} \mathrm{IC}_{50}(\mu \mathrm{M})$ \\
\hline $\mathbf{5}$ & $>100$ & $16.2 \pm 0.06$ & $20.5 \pm 0.07$ & $29.8 \pm 0.13$ & $35.5 \pm 0.10$ \\
$\mathbf{1 0}$ & $30.0 \pm 2.29$ & $65.7 \pm 0.22$ & $>100$ & $>100$ & $38.1 \pm 0.03$
\end{tabular}

${ }^{a} \mathrm{IC}_{50}$ values determined by non-linear regression of at least three experiments (see ESI, $\uparrow$ pg 13). Errors are the transformed greater extreme of the standard error.

utilise a chemical array $(0.04 \%)^{32}$ and high-throughput screen $(0.07 \%) .^{33}$ Applying peptide directed binding to the p53- $h \mathrm{DM} 2 / \mathrm{X}$ PPIs has identified new scaffolds for modulating these PPIs, which have excellent lead like features. ${ }^{34}$ Importantly, this process has identified selective inhibitors for both $h \mathrm{DM} 2$ and $h$ DMX, despite the high structural homology of the binding sites of these proteins. Only two small molecules have been previously reported which selectively modulate the p53- $h \mathrm{DMX}$ PPI, highlighting the power of this technique. Virtual peptide directed binding has identified compounds that interact with the p53 binding site of $h \mathrm{DM} 2$ and $h \mathrm{DMX}$, supported by activity in competitive fluorescence anisotropy assays and, more significantly, quantitative STD NMR analysis. A subset of the identified modulators was shown to have activity towards cancer cell lines that are known to have dependence on $h \mathrm{DM} 2$, $h \mathrm{DMX}$ or both, and these compounds were shown to induce p53 activation downstream events.

\section{Discussion}

The deployment of in silico peptide directed binding has demonstrated an extraordinarily high hit rate for new PPI small molecule modulators of the p53 $h \mathrm{DM} 2 / \mathrm{X}$ interaction. Perhaps more interestingly, this process has identified new binding motifs for these PPIs, as well as highlighting selective scaffolds for $h \mathrm{DMX}$, a notoriously challenging task. ${ }^{35,36}$ The nature of peptide directed binding provides structure activity relationship information without producing specific modifications. The fluoroaryl group shows enhanced binding to $h \mathrm{DMX}$, and the 2,4difluoroaryl compound may offer selectivity for $h \mathrm{DMX}$ (i.e. 5 and
11). The Fmoc azoalanine drives binding to $h \mathrm{DM} 2$, a phenomenon we have shown previously, ${ }^{13}$ but does not guarantee binding (S1 - see ESI, $\dagger$ pg. 9). The presence of the sugar moiety imparts potent binding, but again does not guarantee it (S8). Despite the FP and DSF results supporting tight binding, the hydrophilic sugar moiety raises concerns about compounds 2 and 3 as leads, as the p53 binding site is known to be hydrophobic. Docking suggested the polar sugar occupying the Phe binding pocket of $\mathbf{1}$ (see ESI, $\dagger$ pg 14), but docking results should be interpreted with caution. Modifying the sugar with nonpolar protecting groups reduces potency, but affinity is still observed (i.e. 10 and 11), suggesting key binding moieties may still be available. Investigation of $\mathbf{2}$ and $\mathbf{3}$ as leads is ongoing. The in silico calculations highlighted the zidovudine structure (azide section of 9) as a likely potent binder, but experimentally resulted in a poor hit rate, perhaps highlighting modifications to be made in compound selection process or docking calculations (see ESI, $\dagger$ pg 9).

The compounds identified which selectively modulate the p53- $h$ DMX interaction outperform the two selective molecules reported in the literature. The first known selective binder of $h \mathrm{DMX}$ - SJ-172550 - was demonstrated to bind with less affinity to $h \mathrm{DMX}$ than p53. Both $\mathbf{5}$ and $\mathbf{1 1}$ demonstrate greater binding affinity to $h$ DMX than p53 (ESI, $\dagger$ pg 10). ${ }^{35}$ Additionally, the cellular activity of these compounds is reported in the millimolar range, with our compounds demonstrating at least two orders of magnitude improvement. SJ-172550 is also known to not be a competitive binder, but rather induces a conformational change through covalent bond formation, which does not
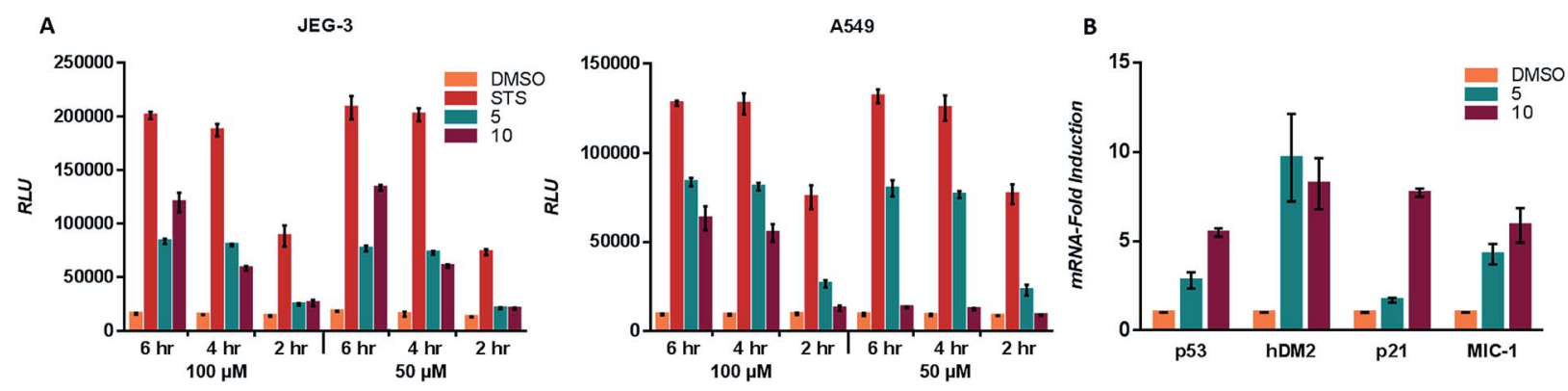

Fig. 4 (A) A549 and JEG-3 cells were treated with vehicle (DMSO), positive control (staurosporine, STS), 5 and 10 at $100 \mu \mathrm{M}$ and $50 \mu \mathrm{M}$ for $6 \mathrm{~h}, 4 \mathrm{~h}$ and $2 \mathrm{~h}$ at $37^{\circ} \mathrm{C}$. Treatment with Caspase-Glo 3/7 reagent (Promega) allowed for the monitoring of the cleavage of pro-luminescent substrate by caspase 3/7. Data are mean \pm SEM for at least $n=3$. RLU - relative luminescent units. STS - staurosporine. (B) JEG-3 cells were treated with vehicle (DMSO), 5 and 10 at $100 \mu \mathrm{M}$ for $6 \mathrm{~h}$ at $37^{\circ} \mathrm{C}$, and transcriptional upregulation of p53, hDM2, p21 and MIC-1 was evaluated by qPCR analysis. $18 \mathrm{~S}$ was used as a control. $Y$-axis is the mRNA-fold induction vs. the vehicle control (DMSO). All $p$ values were less than 0.05 when compared to the vehicle. 
exist in a reducing environment. ${ }^{37}$ Unfortunately, this limits the value of SJ-172550 as a lead candidate.

CTX1, the second small molecule shown to have selectivity for $h \mathrm{DMX}$, has not been validated in in vitro protein assays, with this compound being identified through a cellular screen. ${ }^{9}$ CTX1 demonstrated $\mathrm{IC}_{50}$ values in the tens to hundreds of micromolar range. Compound $\mathbf{5}$ possesses slightly improved potency, but has other important advantages. CTX1 is an acridine based molecule, known to bind to DNA, and act as a PAIN molecule. This characteristic makes CTX1 unusable in many light based assays, such as fluorescence, due to interference. It is also unknown whether CTX1 is a competitive inhibitor of p53 or acts through some other mechanism (some suggestion the CTX1 mode of action overlaps with the action of 9-aminoacridine), ${ }^{9}$ making its use as a chemical tool limited.

SJ-172550 was found as one of three hits in a library of 295848 compounds. ${ }^{35}$ CTX1 came from a screen of over 20000 compounds. ${ }^{9}$ In this instance we have prepared twenty molecules and identified two selective p53- $h$ DMX modulators. The true value of the significant advance in the improvement of success rate we have demonstrated here is the power of peptide directed binding to allow researchers in academia and the pharmaceutical industry, chemistry and biology to quickly and cheaply develop modulators for the protein-protein interaction they wish to target. The need for enormous libraries of molecules and expensive high throughput screening facilities is not necessary for the identification of small molecule modulators, as has been the case for so many challenging targets.

Notably, the compounds prepared are unoptimised but still display strong activity in the fluorescence anisotropy assay and low micromolar cellular activity, highlighting the rapid ability of peptide directed binding to identify selective chemical probes.

\section{Conclusions}

This work has exemplified the power of peptide directed binding to rapidly identify small molecule PPI modulators, at a high hit rate, that show selectivity for challenging targets. This method complements experimental peptide directed binding and is expected to be readily applied to other PPIs such as 14-33/tau, bromodomains or Nrf2/Keap1.

\section{Conflicts of interest}

There are no conflicts to declare.

\section{Acknowledgements}

SJW is funded by the Biotechnology and Biological Sciences Research Council (BBSRC) Doctoral Training Partnership PhD studentship. SP is funded by BBSRC grant no BB/N008448/1. We acknowledge the EPSRC UK National Mass Spectrometry Facility at Swansea University. We thank Professor Aart Jochemsen (Leiden) for the kind gift of $h \mathrm{DMX}$.

\section{Notes and references}

1 L. T. Vassilev, J. Med. Chem., 2005, 48, 4491-4499.

2 V. Tisato, R. Voltan, A. Gonelli, P. Secchiero and G. Zauli, J. Hematol. Oncol., 2017, 10, 133.

3 F. Bernal, M. Wade, M. Godes, T. N. Davis, D. G. Whitehead, A. L. Kung, G. M. Wahl and L. D. Walensky, Cancer Cell, 2010, 18, 411-422.

4 V. Böttger, A. Böttger, C. Garcia Echeverria, Y. F. M. Ramos, A. J. van der Eb, A. G. Jochemsen and D. P. Lane, Oncogene, 1999, 18, 189.

5 F. Toledo and G. M. Wahl, Int. J. Biochem. Cell Biol., 2007, 39, 1476-1482.

6 A. Shvarts, W. T. Steegenga, N. Riteco, T. Laar, P. Dekker, M. Bazuine, R. C. Ham, W. H. v. Oordt, G. Hateboer, A. J. Eb and A. G. Jochemsen, EMBO J., 1996, 15, 5349-5357. 7 Y. S. Chang, B. Graves, V. Guerlavais, C. Tovar, K. Packman, K.-H. To, K. A. Olson, K. Kesavan, P. Gangurde, A. Mukherjee, T. Baker, K. Darlak, C. Elkin, Z. Filipovic, F. Z. Qureshi, H. Cai, P. Berry, E. Feyfant, X. E. Shi, J. Horstick, D. A. Annis, A. M. Manning, N. Fotouhi, H. Nash, L. T. Vassilev and T. K. Sawyer, Proc. Natl. Acad. Sci., 2013, 110, E3445-E3454.

8 B. Graves, T. Thompson, M. Xia, C. Janson, C. Lukacs, D. Deo, P. Di Lello, D. Fry, C. Garvie, K. S. Huang, L. Gao, C. Tovar, A. Lovey, J. Wanner and L. T. Vassilev, Proc. Natl. Acad. Sci., 2012, 109, 11788-11793.

9 G. Karan, H. Wang, A. Chakrabarti, S. Karan, Z. Liu, Z. Xia, M. Gundluru, S. Moreton, Y. Saunthararajah, M. W. Jackson, M. K. Agarwal and D. N. Wald, Mol. Cancer Ther., 2016, 15, 574-582.

10 S. Datta, M. E. Bucks, D. Koley, P. X. Lim and S. N. Savinov, Bioorg. Med. Chem., 2010, 18, 6099-6108.

11 F. Wachter, A. M. Morgan, M. Godes, R. Mourtada, G. H. Bird and L. D. Walensky, Oncogene, 2017, 36, 2184.

12 G. M. Popowicz, A. Dömling and T. A. Holak, Angew. Chem., Int. Ed., 2011, 50, 2680-2688.

13 M. M. D. Cominetti, S. A. Goffin, E. Raffel, K. D. Turner, J. C. Ramoutar, M. A. O'Connell, L. A. Howell and M. Searcey, Bioorg. Med. Chem. Lett., 2015, 25, 4878-4880.

14 J. P. Plante, T. Burnley, B. Malkova, M. E. Webb, S. L. Warriner, T. A. Edwards and A. J. Wilson, Chem. Commun., 2009, 5091-5093, DOI: 10.1039/B908207G.

15 A. Shaginian, L. R. Whitby, S. Hong, I. Hwang, B. Farooqi, M. Searcey, J. Chen, P. K. Vogt and D. L. Boger, J. Am. Chem. Soc., 2009, 131, 5564-5572.

16 F. Bernal, A. F. Tyler, S. J. Korsmeyer, L. D. Walensky and G. L. Verdine, J. Am. Chem. Soc., 2007, 129, 2456-2457.

17 K. Sakurai, C. Schubert and D. Kahne, J. Am. Chem. Soc., 2006, 128, 11000-11001.

18 J. Kallen, A. Goepfert, A. Blechschmidt, A. Izaac, M. Geiser, G. Tavares, P. Ramage, P. Furet, K. Masuya and J. Lisztwan, J. Biol. Chem., 2009, 284, 8812-8821.

19 A. M. Beekman, M. A. O'Connell and L. A. Howell, Angew. Chem., Int. Ed., 2017, 56, 10446-10450. 
20 K. Zhu, K. W. Borrelli, J. R. Greenwood, T. Day, R. Abel, R. S. Farid and E. Harder, J. Chem. Inf. Model., 2014, 54, 1932-1940.

21 W. Sherman, H. S. Beard and R. Farid, Chem. Biol. Drug Des., 2006, 67, 83-84.

22 B. Hu, D. M. Gilkes and J. Chen, Cancer Res., 2007, 67, 88108817.

23 A. Czarna, G. M. Popowicz, A. Pecak, S. Wolf, G. Dubin and T. A. Holak, Cell Cycle, 2009, 8, 1176-1184.

24 R. J. Steel, M. A. O'Connell and M. Searcey, Bioorg. Med. Chem. Lett., 2018, 28, 2728-2731.

25 F. H. Niesen, H. Berglund and M. Vedadi, Nat. Protoc., 2007, 2, 2212.

26 A. Madhumalar, L. H. Jun, C. J. Brown, D. P. Lane and C. S. Verma, Cell Cycle, 2009, 8, 2828-2836.

27 J. Angulo, S. A. Goffin, D. Gandhi, M. Searcey and L. A. Howell, Chem.-Eur. J., 2016, 22, 5858-5862.

28 B. Bertrand, J. Fernandez Cestau, J. Angulo, M. M. D. Cominetti, Z. A. E. Waller, M. Searcey, M. A. O'Connell and M. Bochmann, Inorg. Chem., 2017, 56, 5728-5740.

29 J. Angulo, I. Díaz, J. J. Reina, G. Tabarani, F. Fieschi, J. Rojo and P. M. Nieto, ChemBioChem, 2008, 9, 2225-2227.
30 V. Jayalakshmi and N. R. Krishna, J. Magn. Reson., 2002, 155, 106-118.

31 M. Hirose, K. Yamato, S. Endo, R. Saito, T. Ueno, S. Hirai, H. Suzuki, M. Abei, Y. Natori and I. Hyodo, Oncoscience, 2014, 1, 830-843.

32 T. Noguchi, S. Oishi, K. Honda, Y. Kondoh, T. Saito, T. Kubo, M. Kaneda, H. Ohno, H. Osada and N. Fujii, Bioorg. Med. Chem. Lett., 2013, 23, 3802-3805.

33 L. Gu, H. Zhang, T. Liu, S. Zhou, Y. Du, J. Xiong, S. Yi, C.-K. Qu, H. Fu and M. Zhou, Cancer Cell, 2016, 30, 623-636. 34 D. J. Foley, A. Nelson and S. P. Marsden, Angew. Chem., Int. Ed., 2016, 55, 13650-13657.

35 D. Reed, Y. Shen, A. A. Shelat, L. A. Arnold, A. M. Ferreira, F. Zhu, N. Mills, D. C. Smithson, C. A. Regni, D. Bashford, S. A. Cicero, B. A. Schulman, A. G. Jochemsen, R. K. Guy and M. A. Dyer, J. Biol. Chem., 2010, 285, 10786-10796.

36 M. Wade, Y. C. Li and G. M. Wahl, Nat. Rev. Cancer, 2013, 13, 83.

37 M. Bista, D. Smithson, A. Pecak, G. Salinas, K. Pustelny, J. Min, A. Pirog, K. Finch, M. Zdzalik, B. Waddell, B. Wladyka, S. Kedracka-Krok, M. A. Dyer, G. Dubin and R. K. Guy, PLoS One, 2012, 7, e37518. 